\title{
A NOTE ON THE COMPARISON OF INDUCTANCES, OR OF AN INDUCTANCE AND A CAPACITY BY AN ELECTROMETER METHOD.
}

\author{
By Alva W. Smith.
}

\section{SYNOPSIS.}

A method of comparing inductances is described. This method makes use of an electrometer whose quadrants are connected across a condenser in series with the inductance and whose needle is connected to the middle of a high resistance shunting the condenser and inductance. An equation connecting the deflection of the electrometer with the inductance, capacity and current in the circuit is developed. This equation states that the deflection of the electrometer is proportional to the inductance, to the square of the current and inversely proportional to the capacity. This equation is checked experimentally. By this method several inductances are measured and the results found to be in agreement with those obtained with an A. C. bridge.

IN Fig. I, $L R$ represents a coil of resistance $R$ and inductance $L$ to be measured, $C$ is a variable condenser, $E$ a quadrant electrometer with its pairs of quadrants connected across the condenser and its needle connected to the middle of the high resistance $H R$ (I00,000 ohms), the latter being non-inductive. $A$ is a shunted Duddell thermo-galvanometer used as an ammeter and $G$, an A.C. generator.

Let $v_{1}, v_{2}, v_{3}, v_{4}$ represent the instantaneous values of the potentials at the points indicated on the diagram. The deflection of the electrometer ${ }^{1}$

$$
d=\frac{K}{T} \int_{0}^{T}\left(v_{1}-v_{2}\right)\left(v_{3}-\frac{v_{1}+v_{2}}{2}\right) d t
$$

where $K$ is the electrometer constant and $T$ the period of the alternating current. Since the needle is connected to the middle of $H R$,

$$
\begin{gathered}
v_{3}=\frac{v_{1}+v_{4}}{2} . \\
d=\frac{K}{2 T} \int_{0}^{T}\left(v_{1}-v_{2}\right)\left(v_{4}-v_{2}\right) d t .
\end{gathered}
$$

Assume that a harmonic current

$$
i=I_{m} \sin \omega t
$$

${ }^{1}$ F. M. Laws, Electrical Measurement, p. 250. 
is following in the circuit. Then

$$
\begin{gathered}
v_{1}-v_{2}=\frac{\mathrm{I}}{C} \int i d t=-\frac{I_{m}}{C \omega} \cos \omega t, \\
v_{2}-v_{4}=L \frac{d i}{d t}+R i=L I_{m} \omega \cos \omega t+R I_{m} \sin \omega t . \\
d=\frac{K}{2 T} \int_{0}^{T}\left(\frac{L I_{m}^{2}}{C} \cos ^{2} \omega t+\frac{R I_{m}^{2}}{C \omega} \sin \omega t \cos \omega t\right) d t=\frac{K}{2} \frac{L I_{m}^{2}}{2 C},
\end{gathered}
$$

since average value of $\cos ^{2} \omega t=\mathrm{I} / 2$ and average value of $\sin \omega t \cos \omega t=0$. Expressing the maximum value of the current in terms of the effective value, we have

$$
d=\frac{K}{2} \frac{L I^{2}}{C} .
$$

This equation will hold only for an electrometer obeying the general law of equation ( $\mathrm{I}$ ). It is only the wattless components of the voltage across the condenser and coil that are operative. If, however, the condenser has either leakage or absorption, the deflection will be in part determined by the power components and no longer be expressed by the

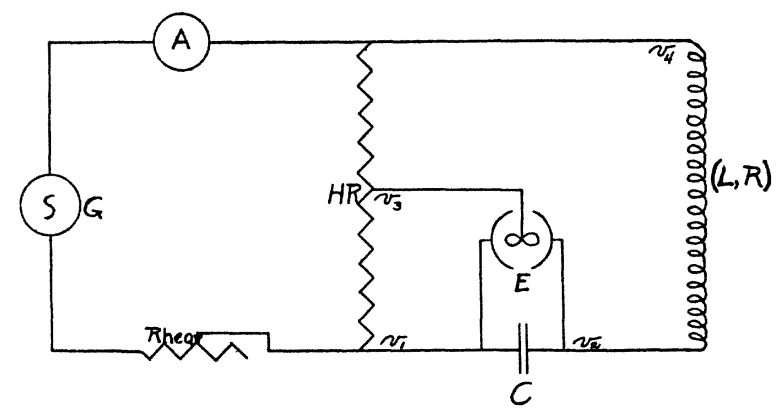

Fig. 1.

simple equation (7). The validity of this equation does not depend upon the assumption of a simple sine wave form. For with a more complex wave form the deflection is

$$
d=\frac{K}{2} \frac{L}{C}\left(I_{1}^{2}+{I_{1}}^{2}+{I_{2}}^{2}+{I_{2}}^{2}+\cdots\right)
$$

where $I_{m}, I_{m}{ }^{\prime}$, are the amplitudes of the sine and of the cosine terms respectively of the $m$ th-harmonic. The parenthesis is the square of the effective current and is equal to $I^{2}$ in equation (7).

To test this equation experimentally, $L, C$ and the frequency were first kept constant and the electrometer deflections read for a series of values of currents. The values of $d$ are plotted against $I^{2}$ in graph (I), 
Fig. 2, and some of the observed values recorded in Table I., (A). The graph shows that the deflections are proportional to the square of the current, if the inductance, capacity and frequency are kept constant.

TABle I.

Tests on $d=\frac{K}{2} \frac{L I^{2}}{C}$.

\begin{tabular}{|c|c|c|c|c|c|}
\hline \multicolumn{2}{|c|}{ (A) I Variable. } & \multicolumn{2}{|c|}{ (B) $C$ Variable. } & \multicolumn{2}{|c|}{ (C) $L$ Variable. } \\
\hline$I$ amps. & $d \mathrm{~cm}$ & $C \mathrm{mf}$. & $d \mathrm{~cm}$. & $L \mathrm{mh}$. & $d \mathrm{~cm}$. \\
\hline .052 & 1.58 & .1 & 9.80 & 7.0 & 2.00 \\
\hline .075 & 3.30 & .133 & 7.35 & 15.0 & 4.22 \\
\hline .095 & 5.14 & .143 & 6.83 & 20.0 & 5.70 \\
\hline .115 & 7.72 & .167 & 5.90 & 30.0 & 8.37 \\
\hline .143 & 11.50 & .2 & 4.95 & 40.0 & 11.19 \\
\hline .172 & 16.35 & .25 & 3.90 & 50.0 & 13.97 \\
\hline .197 & 21.5 & .3 & 3.25 & 60.0 & 16.77 \\
\hline .218 & 26.35 & .7 & 1.39 & 70.0 & 19.47 \\
\hline .223 & 27.6 & .9 & 1.10 & 75.0 & 20.97 \\
\hline \multicolumn{2}{|c|}{$\begin{array}{l}L=50 \mathrm{mh} \\
C=1 \mathrm{mf} \\
f=590 \mathrm{cycles} / \mathrm{sec}\end{array}$} & \multicolumn{2}{|c|}{$\begin{aligned} L & =75 \mathrm{mh} \\
I & =.150 \mathrm{amps} \\
f & =620 \mathrm{cycles} / \mathrm{sec}\end{aligned}$} & \multicolumn{2}{|c|}{$\begin{array}{l}I=.125 \text { amps. } \\
C=1 \mathrm{mf} \\
f=650 \text { cycles } / \mathrm{sec}\end{array}$} \\
\hline
\end{tabular}

Graph (2), Fig. 2, is one obtained by keeping $L, I$ and $C$ constant and varying the frequency. Deflections of the electrometer are plotted

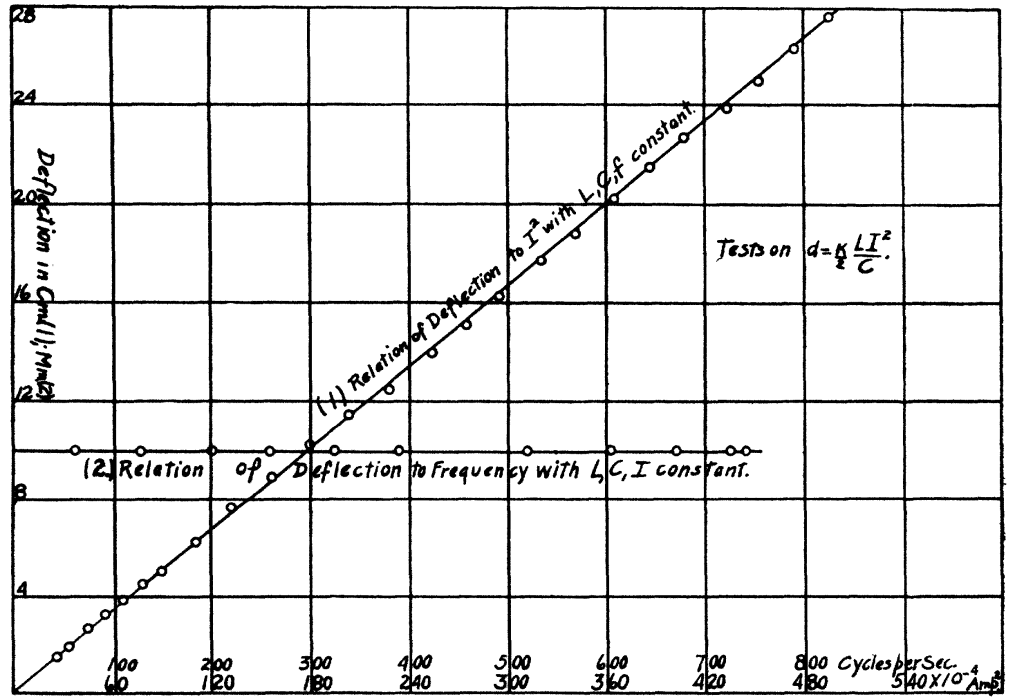

Fig. 2. 
against frequency. It shows that the deflections are independent of the frequency over the range of frequencies used.

A further test was made by keeping $L, I$ and the frequency constant and varying $C$. In graph (3) of Fig. 3 are shown the results obtained. Deflections are plotted against the reciprocals of the capacity. Proportionality between these two variables is shown here.

Tests were then made with $I, C$ and the frequency constant and $L$ variable. Graphs (4), (5), (6) were obtained, different values of current being used. The foregoing graphs confirm equation (7).

In measuring an inductance of unknown value, the deflection of the electrometer is observed with this inductance in circuit and $I$ and $C$ adjusted to give suitable electrometer deflections. An inductance stand-

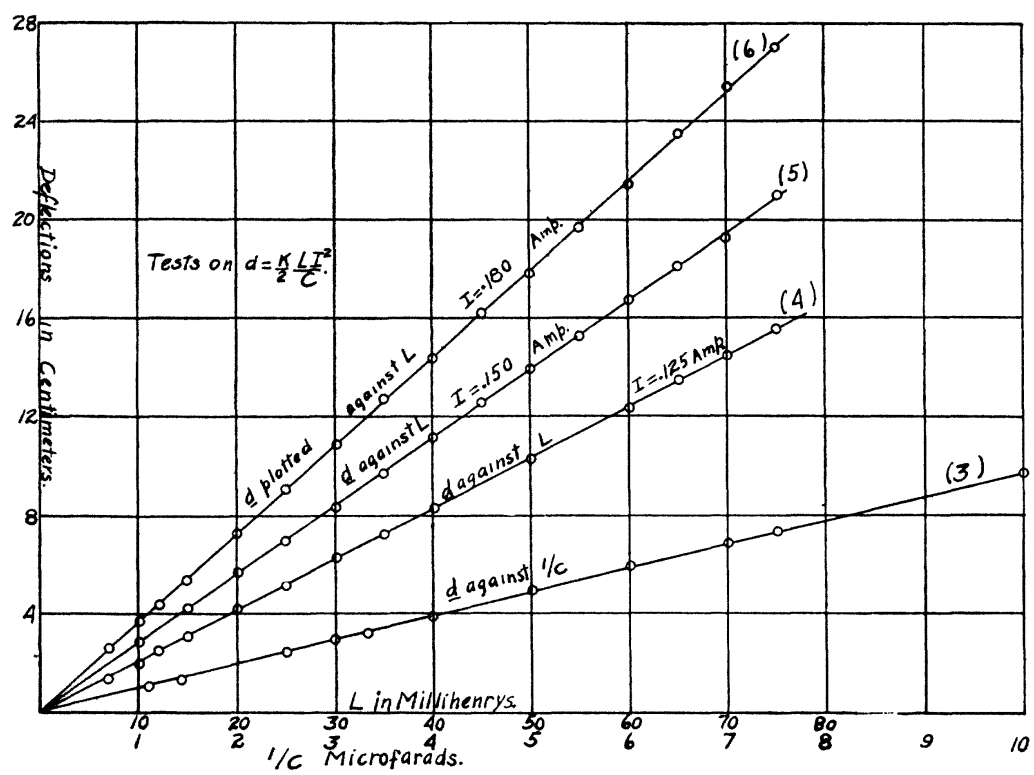

Fig. 3.

ard is substituted for the unknown, and the deflection read for the same value of current and capacity. The inductances then are proportional to their respective deflections. An alternative solution is to determine the electrometer constant $K$ and apply equation ( 7 ) for any convenient values of current and capacity.

Measurements by this method have been compared with those given by the Maxwell bridge method. From such a comparison Table II. is compiled. In this table $L$ is the value of inductance measured by the bridge method and $L^{\prime}$ is its value measured by the electrometer method. 
The average deviation between the values given by the two methods is about .5 per cent.

TABLE II.

\begin{tabular}{c|c|c|c}
\hline Coil. & $L$ in $m h$. & $L^{\prime}$ in $m h$. & Per Cent. Diff. \\
\cline { 2 - 4 } 1 & 19.97 & 20.00 & .2 \\
2 & 94.4 & 94.6 & .2 \\
3 & 110.4 & 110.0 & .4 \\
4 & 128.0 & 127.0 & .8 \\
5 & 154.8 & 154.5 & .2 \\
6 & 214.0 & 216.0 & .9 \\
7 & 318.0 & 321.0 & .95 \\
\hline
\end{tabular}

For given values of current, capacity and inductance these methods appear equally sensitive for all frequencies used. In this respect it seems superior to methods involving the use of a telephone receiver or a vibration galvanometer as a detector. Moreover, it does not involve a knowledge of the frequency as is required in the Wilson method. ${ }^{1}$ Of course, for a given impressed voltage, that frequency which coincides most nearly with the resonant frequency of the circuit containing $L$ and $C$ will give the maximum current and hence maximum electrometer deflection, thus making the sensibility of the method a maximum.

Physical Laboratory,

Ohio State University.

${ }^{1}$ F. M. Laws, Electrical Measurements, p. 4I4. 